\title{
A Process Model for Generating and Evaluating Ideas: The Use of Machine Learning and Visual Analytics to Support Idea Mining
}

\author{
Workneh Y. Ayele ${ }^{(\bowtie)}(1)$ and Gustaf Juell-Skielse (1) \\ Department of Computer and Systems Sciences, Stockholm University, Stockholm, Sweden
}

\begin{abstract}
The significance and possibilities of idea generation and evaluation are increasing due to the increasing demands for digital innovation and the abundance of textual data. Textual data such as social media, publications, patents, documents, etc. are used to generate ideas, yet manual analysis is affected by bias and subjectivity. Machine learning and visual analytics tools could be used to support idea generation and evaluation, referred to as idea mining, to unlock the potential of voluminous textual data. Idea mining is applied to support the extraction of useful information from textual data. However, existing literature merely focuses on the outcome and overlooks structuring and standardizing the process itself. In this paper, to support idea mining, we designed a model following design science research, which overlaps with the Cross-Industry-Standard-Process for Data Mining (CRISP-DM) process and adapts well-established models for technology scouting. The proposed model separates the duties of actors in idea mining into two layers. The first layer presents the business layer, where tasks performed by technology scouts, incubators, accelerators, consultants, and contest managers are detailed. The second layer presents the technical layer where tasks performed by data scientists, data engineers, and similar experts are detailed overlapping with CRISP-DM. For future research, we suggest an ex-post evaluation and customization of the model to other techniques of idea mining.
\end{abstract}

Keywords: Idea mining - Idea generation · Idea evaluation · Text mining · Machine learning · Dynamic topic modeling

\section{Introduction}

The importance and possibilities of idea generation and evaluation are growing due to the increasing demands for digital innovation and the abundance of data. The accumulation of large data repositories, such as patents, could inspire people to discover and accelerate innovation from solutions to similar problems $[1,2]$. Private sector organizations, as well as governmental organizations, could benefit from open and closed data repositories through extracting useful information. In this paper, we define idea mining as the process of generating and evaluating ideas from textual data using techniques of machine learning, text mining, NLP, statistics [3], information retrieval [4], and bibliometrics [5]. Idea mining could be used for supporting decision making as part of a decision support system [6]. 
Idea generation and evaluation are parts of cognitive processes of creativity [7], where idea generation is analogous to medicine prescription [8]. Solutions to problems could be used to conjecture solutions to analogous problems [1,2]. The early detection of solutions from patents and publications, which is referred to as technology scouting, reduces the time-lag between the advancement of technology and its discovery [9]. Hence, the process of technology scouting could be used to find solutions to unsolved problems. Besides, ideas could be generated from networks of experts [10], scholarly literature, patents, reports, the Internet, documents [3], social media [11], and crowdsourcing [12].

Academia produces publications in large volumes [13]. Also, academia-industry collaboration nurtures the processes of innovation [14]. However, academia and the industry have different goals; the industry strives to solve problems while academia focuses on knowledge creation [15]. It is beneficial for the industry to explore and exploit the landscape of academia and its knowledge generation activities globally. Yet, it is becoming harder to find innovative ideas despite a swift growth in research findings [13]. Similarly, user-generated social media data is also growing at an unprecedented volume and speed making it hardly possible to analyze the data manually to meet companies' demands [11]. Moreover, manual analysis of scholarly literature is prone to subjectivity and bias [16]. Thus, it is natural to look for alternatives for dealing with overwhelmingly large volumes of data.

Therefore, in the wake of increasing data production and the increasing popularity of machine learning techniques, idea generation and evaluation could be done using data mining techniques. Changes in technology affect the way idea generation is performed [17]. For example, a machine learning technique, such as topic modeling, is used to elicit insights and evaluate the significance of ideas [18]. Also, unsupervised clustering of text, such as Latent Dirichlet Allocation (LDA) and Dynamic Topic Modelling (DTM) techniques (c.f. [19]), can be used to process large volumes of data. Besides, it is beneficial to follow standardized process models, such as Cross Industry Standard Process for Data Mining (CRISP-DM) to ensure reusability. Furthermore, CRISP-DM is independent of the technology used, and industry sector [20] and could be adapted for analyzing unstructured data such as social media data [21].

However, previous works related to idea mining do not integrate and map their models with the well-established CRISP-DM process model, as illustrated in [22]. Yet the work by [22] adapts CRISP-DM for Idea Mining (CRISP-IM), focusing on the task and perspectives of data scientists and data engineers. Besides, existing process models for idea mining use workflows, non-standard diagrams, and standard text mining [22]. For example, [4] used a similar measuring technique, distance measure, to identify solutions to problems. The search query and new textual corpora are used as inputs, and useful new ideas are extracted by calculating the similarity between the query phrase and potential phrases expressing solutions from the corpora [4]. Therefore, the goal of this paper is to propose the IGE (Idea Generation and Evaluation)-model as an extension of the CRISP-IM [22] for supporting idea mining using patterns generated from DTMs, and visual analytics tools.

The IGE-model is designed following design science research and is demonstrated through the evolution of topics, extracted from DTM, unsupervised machine learning [23], and visual analytics applied on scholarly articles [24]. The proposed model has 
two layers, a business layer, and a technical layer. The design and development were iteratively done by using 19 experts' feedback. Also, the proposed model's components are inspired by well-founded models, namely the most widely accepted process model, CRISP-DM [20], and the technology scouting process model [9], other idea evaluation techniques, presented in the next chapter, are also the foundations of the IGE-model. This paper has five chapters: Background and Previous Research, Methodology, Result, Discussion and Future Research, and finally, Conclusions.

\section{Background and Previous Research}

In this section, we briefly present key concepts discussed in this paper, such as "idea," sources of ideas, idea generation, idea evaluation, and finally, related research. An idea is an abstract term that is open to interpretations. On the other hand, idea mining can be used to support decisions in decision support systems [6]. Thorleuchter et al. referred to ideas as a piece of new and useful text phrase consisting of domain-specific terms from the context of technological language usage rather than unstandardized colloquial language [4]. Also, [25] defined an idea as a pair of problem-solution. In this paper, we define idea as: "a sentence or text phrase describing novel and useful information through expressing possible solution(s) to current problems".

Idea Generation. Essential sources of ideas, from which innovation could be achieved, are networks of experts [10], scholarly literature, patents [3, 26], social media [11, 27], Internet, reports, documents [3], and crowdsourcing [12]. Idea generation could be done through collaborating of persons aided by machine learning techniques [12], by finding solutions to analogous problems using machine learning on textual data [1,2], network analysis and text mining [12], algorithms of recommendation systems [25]. Also, idea generation improves idea evaluation as part of forecasting [28].

Idea Evaluation. According to [29] idea evaluation processes consists of hierarchies of evaluation criteria with corresponding attributes such as technical (productivity, functionality, reliability, safety, ecologically, and aesthetics), customer (necessity, novelty, usefulness, and usability), market (competition, buyer, market), financial (sales volume, rate of return, and payback time), and social (importance, emphasis, commitment, and affordability). On the other hand, [30] proposed an idea evaluation hierarchy as four criteria with corresponding attributes. The criteria with corresponding attributes are novelty (originality and paradigm relatedness), workability (acceptability and implementability), relevance (applicability and effectiveness), and specificity (implicational explicitness, completeness, and clarity). An idea can be evaluated using the connectivity of a network of idea providers [10].

It is possible to evaluate ideas using Analytic Hierarchy Process (AHP), Simple Additive Weighting (SAW), and idea evaluation criteria [29]. AHP is a well-known decision-making tool that can be applied when multiple objectives are involved in judging alternatives [31]. Besides, SAW is a decision-making technique developed to solve the subjectivity of personal selection process using multi-criteria selecting [32]. Machine learning could also be used to support idea evaluation. On the other hand, [18] used topic modeling to assess the significance of ideas based on insights. 


\subsection{Text Mining and Natural Language Processing}

Text mining is a subfield of computer science that combines techniques of Natural Language Processing (NLP), knowledge management, data mining, information retrieval, and machine learning [33]. Moreover, the techniques and tools used in text mining are used to analyze social media textual data for commercial and research purposes [34]. Natural Language Processing (NLP) covers any natural language manipulation using computers [35]. NLP can use machine learning for linguistic analysis [36].

Dynamic Topic Modeling. Capturing emerging trends is also possible using topic modeling [37]. Topic modeling is an evolving technique in machine learning which enables the identification of hidden topics from collections of textual datasets [19]. There is an increasingly growing collection of scholarly articles which demands tools such as machine learning technique to analyze [38]. DTM based on LDA is used to elicit the evolution of topics from textual data [39]. LDA is a topic modeling algorithm designed to identify latent topics without temporal patterns [40]. DTM uses multinomial distributions to represents topics. Furthermore, to infer hidden topics, DTM uses Kalman filters, vibrational approximation, and non-parametric wavelet regression [39]. DTM techniques are used to elicit and capture emerging trends [37].

\subsection{Visual Analytics}

According to [41], visual analytics combines a set of techniques such as automated analysis and interactive visualizations. Visual analytics is used for creating an adequate understanding, reasoning, and decision making using large and complex datasets. Also, visual analytics applied to scholarly articles enables elicitation of trends and identification of critical evidence in a flexible, repeatable, timely, and valuable way [42].

\subsection{Related Research}

In this section, examples of research related to computer-assisted idea generation and evaluation are presented. Thorleuchter et al. proposed a method for generating ideas using Euclidean distance measure, and the process model illustrated in their study is merely a general workflow highlighting significant steps done to achieve the desired result [4]. On the other hand, [11] proposed a process model with a scope of knowledge management, using business process modeling notation, for idea generation using social media data, and user participation. Liu et al. applied algorithms that are common in recommendation systems, such as collaborative filtering, to elicit ideas and described the process using a generic workflow [25]. Kao et al. used a simple diagram of idea mining architecture [27]. Authors proposed an idea generation framework at an abstract level using similarity measures $[3,43]$.

It is also possible to evaluate ideas using machine learning, text mining, NLP, and similar techniques [3]. For example, topic modeling techniques such as Latent Semantic Analysis (LSA), which is unsupervised machine learning, is used to predict the relevance of ideas and provide insight [18]. Alksher et al. used a general workflow for idea evaluation process consisting of data preparation, human judgment, statistical analysis, 
reliability testing, and idea evaluation [43]. An idea evaluation for contests using expert judgment, machine learning, and quality criteria was proposed by [44].

In this paper, we propose a model which combines machine learning and domain experts' judgment, and machine learning similar to [44]. CRISP-DM is a standardized process model that is technology and industry sector independent [20]. Our proposed model is also technology and sector independent and built on top of CRISP-DM.

\section{Methodology}

The design science approach followed in this paper consists of six activities, such as identification of problems, objectives of the solution, design and development, demonstration, evaluation, and communication [45]. Running examples and motivations for the design of the model are carried out by demonstration through DTM and visual analytics, experts' semi-structured interviews, and a literature review.

The list of respondents is presented in Table 1. The respondents were chosen based on their expertise. The IGE-model is designed for innovation agents, data and knowledge engineers, innovation accelerators and incubators, and startups dedicated to extracting useful and new ideas. Therefore, two categories of respondents were chosen. The first category of respondents consisting of all respondents (R1-R19) except R6 and R10 are business-related experts dedicated to idea generation, innovation incubation, innovation acceleration, and state-sponsored innovation catalysts. The second category of experts (R5, R6, R10, R16, R17, and R18) are professionals in data visualization and machine learning. Respondents R5, R16, R17, and R18 belong to both categories. The literature review is conducted to motivate the validity of the design, identify techniques of idea mining and corresponding processes.

\subsection{Problem Identification}

Problem identification was conducted using a literature review and semi-structured interviews. The thematic analysis of the selected literature and the interviews, indicated that textual data such as scholarly literature, social media data, and patents, are growing in unprecedented volume, so the manual analysis is infeasible [11, 16]. Respondents (R1-R7) suggested that it is important to support idea generation and evaluation using relevant textual data sources.

\subsection{Objectives of the Solution}

The proposed model, The IGE-model, aims to improve the ability to generate and evaluate ideas from, so far, untapped sources of knowledge. The IGE-model has two objectives elicited through a literature review and semi-structured interviews (R1-R19). The users of the IGE-model could be technology scouts, incubators, accelerators, data mining project managers, and technology innovation actors. The objectives are (1) to support users in generating and evaluating ideas through the use of machine learning, NLP, and similar techniques from textual data, and (2) to serve users as a guideline in structuring the task of idea generation and evaluation form textual data. 
Table 1. List of respondents, where PI stands for: - Problem Identification, PD-stands for: Problem Identification and Design and Development.

\begin{tabular}{l|l|l|l}
\hline$\#$ & $\begin{array}{l}\text { Respondent's organization and } \\
\text { responsibility }\end{array}$ & $\#$ & $\begin{array}{l}\text { Respondent's organization and } \\
\text { responsibility }\end{array}$ \\
\hline R1 & Xlab, Hub manager (PI) & R11 & ATEA, Tech scout (PD) \\
\hline R2 & $\begin{array}{l}\text { Xlab, Community development } \\
\text { (PI) }\end{array}$ & R12 & $\begin{array}{l}\text { Syntesia, CEO \& Founder in } \\
\text { Innovation and Digitization (PD) }\end{array}$ \\
\hline R3 & Bluemoon, Strategy leader (PI) & R13 & $\begin{array}{l}\text { RISE, Västra Götlandsregionen, } \\
\text { Sustainability and innovation expert } \\
\text { (PD) }\end{array}$ \\
\hline R4 & Bluemoon, Lead engineer (PI) & R14 & $\begin{array}{l}\text { Sahlgrenska Science Park, Business } \\
\text { Advisor (PD) }\end{array}$ \\
\hline R6 & $\begin{array}{l}\text { ITM, Innovation fund } \\
\text { administrator (PI) }\end{array}$ & R15 & $\begin{array}{l}\text { Sahlgrenska Science Park, Machine } \\
\text { learning expert (PD) }\end{array}$ \\
\hline R7 & ITM, Incubation manager (PI) & R17 & eGovlab, SU, Visualization expert (PD) \\
\hline R8 & $\begin{array}{l}\text { SU, Idea and business advisor } \\
\text { (PD) }\end{array}$ & R18 & $\begin{array}{l}\text { AAU, iCog, Machine learning, } \\
\text { Evaluated an Incubation Process (PD) }\end{array}$ \\
\hline R9 & $\begin{array}{l}\text { RISE, Digital innovation expert } \\
\text { (PD) }\end{array}$ & R19 & VITALIS, Strategic Advisor (PD) \\
\hline R10 & ATEA, Business analyst (PD) & & \\
\hline & Rounder (PI) & & \\
\hline
\end{tabular}

\subsection{Design and Development, and Demonstration}

The design has been informed by an established method of technology scouting [9], CRISP-DM [20], idea evaluation methods [29], and incremental development through expert feedback (R1-R19). The motivation of the relevance and the validity of the design of the IGE-model is done through a literature review, justifications of adapted models, informed demonstration of DTM using scholarly articles [23], through visual analytics of scholarly articles [24], and through 19 interviews.

\subsection{Evaluation, and Communication}

The ex-post evaluation of the IGE-model is left for future study. The IGE-model is communicated to business analysts, machine learning experts, decision scientists, incubators, accelerators, technology innovation agents, and public innovation centers through workshops and meetings. Also, the model will be publications. 


\section{Result}

The IGE-model has four major phases, see Fig. 1. All phases of the IGE-model, are completely mapped with the CRISP-IM model, as illustrated in Fig. 2 below. The IGE-model is designed to structure the identification of new ideas about technological innovation and to evaluate elicited ideas. The IGE-model separates the duties of business and technical experts while the CRISP-IM serves technical experts. Furthermore, the original model was different from the model presented in this paper. R17 suggested restructuring the model to make it more understandable and usable; More specifically, R17 recommended that idea evaluation should come after idea generation. All respondents accepted the components of the model. Additionally, evaluation criteria were added, as illustrated in Fig. 3 in dotted boxes.

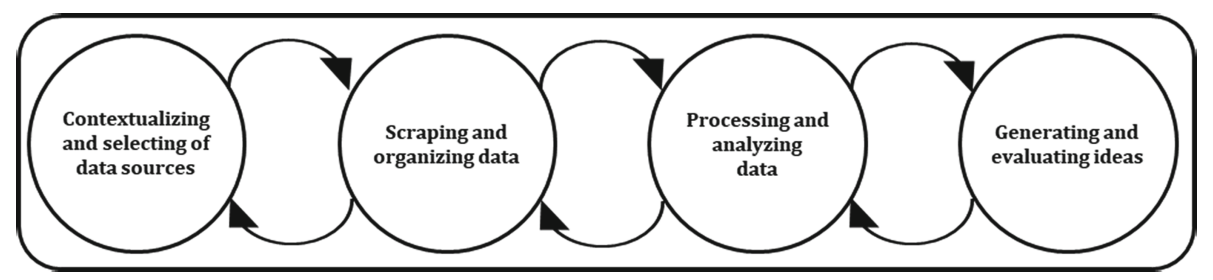

Fig. 1. IGE-model, for generating and evaluating ideas.

The description of each phase and corresponding components are described in Table 2 below. In addition to Table 2, a graphical description of the components of each phase and the illustration of the mapping of the IGE-model with the CRISP-DM model is illustrated in Fig. 2. 
Table 2. Description of phases of the IGE-model

\begin{tabular}{|c|c|c|c|c|}
\hline Phases & $\begin{array}{l}\text { Contextualizing and } \\
\text { selection of data } \\
\text { sources }\end{array}$ & $\begin{array}{l}\text { Scrapping and } \\
\text { organizing data }\end{array}$ & $\begin{array}{l}\text { Processing and } \\
\text { analyzing data }\end{array}$ & $\begin{array}{l}\text { Generating and } \\
\text { evaluating ideas }\end{array}$ \\
\hline Inputs & $\begin{array}{l}\text { - Domain knowledge } \\
\text { - Need for innovation } \\
\text { and problems } \\
\text { - Ideas from other } \\
\text { sources e.g. } \\
\text { contests, scouts, etc. }\end{array}$ & $\begin{array}{l}\text { - Domain knowledge } \\
\text { - Identified goals, } \\
\text { search area, data } \\
\text { sources, and } \\
\text { articulated problem } \\
\text { statement }\end{array}$ & $\begin{array}{l}\text { Domain knowledge } \\
\text { in idea mining, } \\
\text { machine learning, } \\
\text { NLP, etc. } \\
\text { - Organized datasets }\end{array}$ & $\begin{array}{l}\text { - Domain knowledge } \\
\text { - Identified trends, } \\
\text { insights, foresight, } \\
\text { patterns, } \\
\text { visualizations, other } \\
\text { sources of ideas }\end{array}$ \\
\hline Activities & $\begin{array}{l}\text { - Preparation } \\
\text { - Articulate problem } \\
\text { statement } \\
\text { - Define goals } \\
\text { - Identify and select } \\
\text { search area and data } \\
\text { sources, e.g. } \\
\text { scholarly articles, } \\
\text { journals, patents, } \\
\text { social media data, } \\
\text { etc. }\end{array}$ & $\begin{array}{l}\text { - Formulate search } \\
\text { query using } \\
\text { identified goals and } \\
\text { problem statements } \\
\text { - Scrape and organize } \\
\text { data } \\
\text { - Based on the quality } \\
\text { and quantity of data } \\
\text { make decision to go } \\
\text { back or forward }\end{array}$ & $\begin{array}{l}\text { - Identify tools } \\
\text { - Preprocessing of } \\
\text { data, identification } \\
\text { of trends, patterns, } \\
\text { insights, \& foresight } \\
\text { - Statistical testing } \\
\text { - Evaluating the result } \\
\text { - Use statistical } \\
\text { indicators to go back } \\
\text { or forward }\end{array}$ & 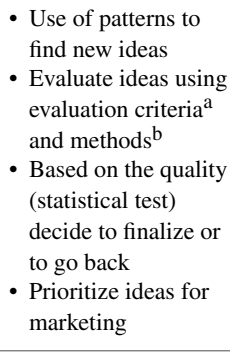 \\
\hline Outputs & $\begin{array}{l}\text { - Defined goals } \\
\text { - Articulated problem } \\
\text { statements } \\
\text { - Identified search } \\
\text { area and data } \\
\text { sources }\end{array}$ & - Organized dataset & $\begin{array}{l}\text { - Final dataset } \\
\text { - Trends, insight, } \\
\text { foresight, } \\
\text { visualization of } \\
\text { correlation and } \\
\text { citation analysis }\end{array}$ & $\begin{array}{l}\text { - Generated ideas } \\
\text { - Prioritized list of } \\
\text { ideas based on } \\
\text { evaluation } \\
\text { - Best practices, } \\
\text { Domain knowledge }\end{array}$ \\
\hline Evaluation of Phases & $\begin{array}{l}\text { Relevance of goals, } \\
\text { search area, and } \\
\text { selection of data } \\
\text { sources }\end{array}$ & $\begin{array}{l}\text { The relevance, } \\
\text { adequacy, and } \\
\text { quality of collected } \\
\text { data }\end{array}$ & $\begin{array}{l}\text { Quality of trends } \\
\text { and patterns based } \\
\text { on interpretability } \\
\text { and statistical tests }\end{array}$ & $\begin{array}{l}\text { Number and } \\
\text { percentage of viable } \\
\text { ideas identified }\end{array}$ \\
\hline
\end{tabular}

${ }^{\mathrm{a}}$ The evaluation of ideas, as suggested by [29] can be done by the rating of criteria and for additional attributes elicited from experts and literature, refer to Fig. 3 below.

${ }^{\mathrm{b}}$ The rating of idea efficacy attributes can be done using Simple Additive Weighting (SAW) [32] or Analytical Hierarchy Process (AHP) method [31].

\subsection{Phase 1: Contextualizing and Selection of Data Sources}

In this phase, domain knowledge, the need for innovation, and commercialization are used as inputs. Additionally, idea banks, ideas obtained from contests, ideas from scouts through formal sources (conferences, technology bulletins, etc.) and informal sources (networks of experts) are also inputs. The activities in this phase are defining goals, articulating problem statements, identifying search areas, identifying, and selecting formal data sources, e.g., scientific articles, journals, patents, social media data, etc. The outputs of this phase are defined goals, identified search area, identified data sources, and articulated problem statement.

Motivation - Defining goals and search areas, and selection of data sources are parts of technology scouting process [9]. Business understanding, which mainly focuses on technology need assessment, is the first stage of the CRISP-DM process model [20]. Also, R9 suggested preparation and planning to be added in the first phase of idea generation and evaluation. 


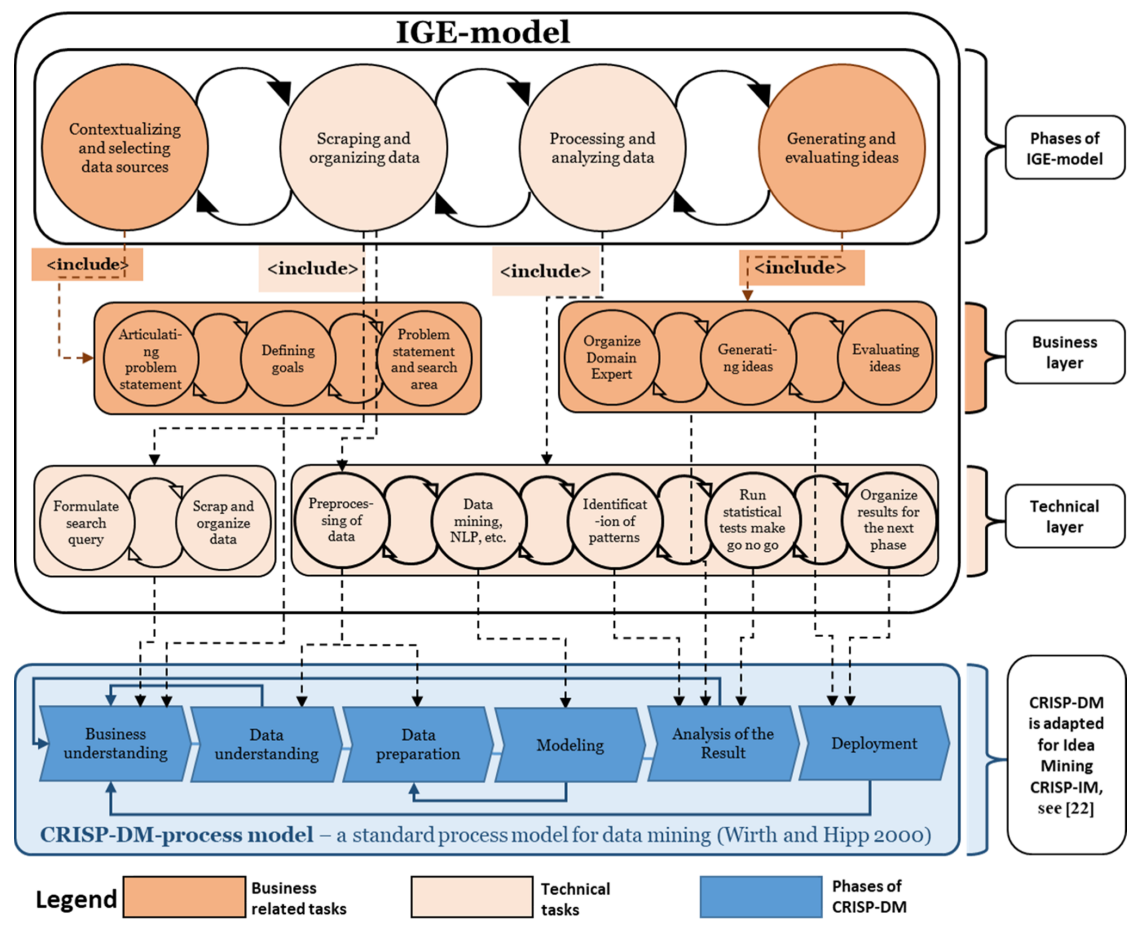

Fig. 2. IGE-model, illustrating business and technical layers, and the mapping of the IGE-model with CRISP-DM (the CRISP-DM is adapted for idea mining CRISP-IM [22], pp. 6).

Demonstration - Tasks in the process as an illustration [23] were done for the identification of goals and success criteria. Goals identified were - To be able to identify trends, to be able to identify emerging patterns, to be able to use trends to evaluate and generate ideas. Identified resources and the activities were locating datasets such as scientific literature, social media data, web forums, etc.

\subsection{Phase 2: Scraping and Organizing Data}

In this phase, using domain knowledge, identified goals, articulated problem statements, and identified search area search query is articulated. Data cleaning is done by reformatting data into a structured dataset by removing anomalies. Exploring and describing the data are done using Python, spreadsheet applications, etc. Finally, based on the quality and the adequacy of the data, it is decided to use the datasets and continue or go back to the previous phase to redefine the search query.

Motivation - Data collection, understanding, and identification of quality issues are parts of the CRISP-DM phase, data understanding [20]. Also, data collection is part of a technology scouting process [9]. Also, business understanding, which mainly focuses on technology need assessment, is the first stage of the CRISP-DM process model. 
Demonstration - For demonstration purposes, Scopus was chosen for data collection. It is possible to use other data sources. Scopus has updated and larger datasets of scholarly literature than Web of Science [46]. After formulating a final query, a total of 5425 documents were extracted. Duplicates were also removed using reference management tools such as Zotero and Mendeley.

\subsection{Phase 3: Processing and Analyzing Data}

In this phase, using domain knowledge and tools, data is preprocessed to produce the final dataset to be fed to models of machine learning. Statistical testing is used to assess the quality of patterns, trends, insights, and foresight generated. If the statistical tests and evaluations indicate that there are needs to scrape more data, then it is recommended to go back to the previous phase and modify the query and reconsider to include more data sources.

Motivation - Data preparation is the preprocessing of datasets to prepare the final datasets to be fed to the modeling phase of the CRISP-DM [20]. So, the final dataset is prepared in this stage. By using machine learning techniques, it is possible to improve the ability to generate and evaluate the quality of ideas from, so far, untapped sources of knowledge. Machine learning methods, such as LDA and DTM techniques (c.f. [19]), can be used to analyze large numbers of data, for example, academic publications, patent descriptions, and social media, to identify and present topics and their relevance, and indicate the evolution of topics over time.

Demonstration - The dataset was preprocessed using Python. Preprocessing was aided by visualization of term frequency, as illustrated in [23]. After preprocessing, the final dataset was fed to the DTM, for identifying the evolution of topics. Also, trends and patterns were identified through visual analytics of scholarly articles [24]. Visualization of trends, insights, foresight, and patterns were the outputs of this phase.

\subsection{Phase 4: Generating and Evaluating Ideas}

In this last phase, using domain knowledge, identified trends, insights, foresight, and patterns, ideas are generated. Domain experts could decide to use quality attributes to decide to go back to the previous phase or to continue generating, evaluating, and prioritizing ideas. Ideas could be evaluated using a computer-assisted technique or simply using idea evaluation techniques available in the literature. We present available idea efficacy factors as illustrated below, see Fig. 3, but users of this model could pick more appropriate criteria and adapt the suggested criteria.

Motivation - It is possible to generate and evaluate ideas using machine learning techniques. Emerging trends elicited from topics are used for decision making in science and technology [47]. Analysis of trends results in forecasting trends in technology [48], forecasting while idea generation improves idea evaluation [28]. Machine learning, AI, and analytics can be used to elicit ideas by forecasting relevance and extracting valuable 


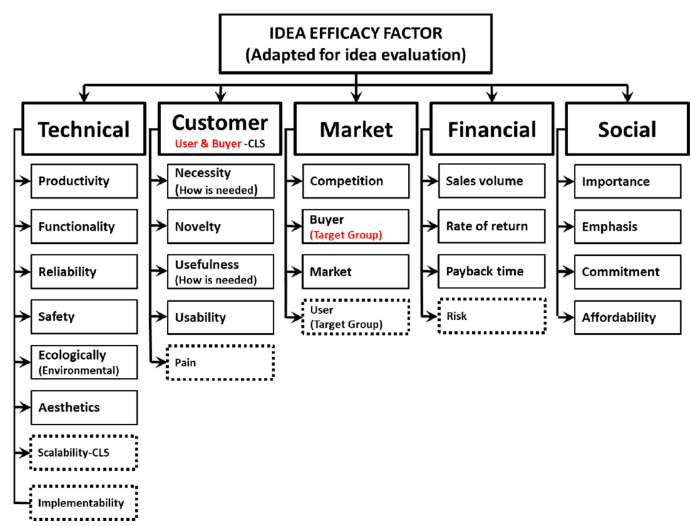

Fig. 3. Adapted idea evaluation criteria [29], criteria in dotted boxes are elicited through semistructured interviews and a literature review.

insights [18]. Similarly, it is possible to elicit trends and identify temporal patterns using machine learning, scientometric, and visual analytics [24]. Also, [44] proposed an idea evaluation procedure for contests using expert judgment, machine learning, and quality standards [44]. Likewise, the IGE-model includes domain knowledge experts as an input in the idea generation and evaluation phases.

Idea evaluation could be done using idea efficacy factors [29]. The rating of the evaluation of each attribute could be done using the SAW method [32] or AHP [31]. Implementability is added by [30]. Risk under financial, target group (user and buyer) under market, scalability under technical, pain under customer, and finally, two types of customer characters (customer user and customer buyer) were suggested by R8. Furthermore, eliciting and analyzing evolving trends can be helpful for decision-makers and stakeholders in academia and the industry [49]. For example, elicitation of topics about emerging trends in science and technology is crucial for making decisions [47]. Trend analysis can be used for forecasting trends in technology [48].

Demonstration - Research agendas and ideas of innovation were identified using trends, insights, foresight, and other inputs [23]. Thematic analysis and visualization were used to elicit and interpret trends. Identified trends can be used to extrapolate ideas by implications, and generate a report. Also, generated ideas can be used for evaluating the relevance and timeliness of ideas being commercialized by incubators, innovators, and R\&Ds. The thematic analysis of evolving topics was done to analyze, elicit trends, and report patterns and themes following [50].

\section{Discussion and Future Direction}

The purpose of this paper is to support idea generation and evaluation by proposing a process model following the design science approach, which could serve as a guideline for structuring the work of idea generation and evaluation. The proposed IGE-model 
abstracts the alignment of managerial and technical levels and maps its phases with CRISP-DM. The model is motivated by the well-founded technology scouting process model [9], in addition to CRISP-DM. Research activities, findings, and publications are increasing at unprecedented volume while it is becoming hard to find innovative ideas [13]. The need for structuring idea mining arises from the growing volume of scholarly articles, patents, social media data, etc. and with the inability of a human being to process this voluminous data without subjectivity and bias [16]. It is also difficult to be competitive in the global market with manual analysis of titanic data [11].

Previous works mainly focus on generating and evaluating ideas. Besides, existing models are merely simple workflows, or general frameworks focused on using classical data mining techniques. For example, [43] presented an idea evaluation process as a generic workflow. Also, [11] suggested a business process modeling notation, for idea generation. The IGE-model includes the use of domain experts' judgment in its critical phases, and it is also suggested that idea evaluation activities could be done using both machine learning and human involvement.

Our proposed model will enable governments and industries to upgrade their organizations to meet future challenges proactively by generating and evaluating ideas about services and products. Besides, the generation of ideas is the impetus to companies' strategic marketing positions. For example, industries could proactively update their visions and goals based on reliable future market potential and product innovation foresight. Industries, technology incubators, and technology accelerators often make decisions about production and marketing on intuition-based subjective strategists. The proposed model has not been tested in practice. Therefore, for the future, we suggest exante and ex-post evaluation, adapt the model to make it applicable to other idea mining techniques.

\section{Conclusions}

Ideas are initiated to solve problems. Existing solutions created to address wellestablished problems can also be repurposed through the identification of analogous solutions to solve new problems. It is also possible to generate ideas using machine learning assisted techniques. Human involvement in the process of creative idea generation is vital. For example, [51] argued that inspiration from out-of-domain actors of innovation engaged in idea generation enables experienced innovators to have highly likely success in idea generation and an increased chance of having innovativeness in generated ideas.

Finally, idea evaluation followed by idea generation can be supported by machine learning and idea mining techniques. The IGE-model could be used to support technology scouts, innovation agents, incubators, technology accelerators, and relevant stakeholders in practice to manage idea generation processes. The use of IGE-model involves users interested in business and technical perspectives. Thus, IGE-model demands multidisciplinary expertise. We feel that the result of this study is a valuable contribution to research, especially to idea mining and idea management, which involves data and knowledge engineering research. 


\section{References}

1. Hope, T., Chan, J., Kittur, A., Shahaf, D.: Accelerating innovation through analogy mining. In: 23rd ACM SIGKDD International Conference on Knowledge Discovery and Data Mining, KDD, Part F1296, pp. 235-243 (2017)

2. Hope, T., Chan, J., Kittur, A., Shahaf, D.: Accelerating innovation through analogy mining. In: 27th International Joint Conference on Artificial Intelligence, IJCAI, pp. 5274-5278 (2018)

3. Alksher, M.A., Azman, A., Yaakob, R., Kadir, R.A., Mohamed, A., Alshari, E.M.: A review of methods for mining idea from text. In: Third International Conference on Information Retrieval and Knowledge Management (CAMP), pp. 88-93. IEEE (2016)

4. Thorleuchter, D., Van den Poel, D., Prinzie, A.: Mining ideas from textual information. Expert Syst. Appl. 37(10), 7182-7188 (2010)

5. Ogawa, T., Kajikawa, Y.: Generating novel research ideas using computational intelligence: a case study involving fuel cells and ammonia synthesis. Technol. Forecast. Soc. Chang. 120, 41-47 (2017)

6. Thorleuchter, D., Van den Poel, D.: Idea mining for web-based weak signal detection. Futures 66, 25-34 (2015)

7. Puccio, G.J., Cabra, J.F.: Idea generation and idea evaluation: cognitive skills and deliberate practices. In: Handbook of Organizational Creativity, pp. 189-215. Academic Press (2012)

8. Smith, G.F.: Idea-generation techniques: a formulary of active ingredients. J. Creat. Behav. 32(2), 107-134 (1998)

9. Rohrbeck, R.: Technology scouting-a case study on the Deutsche Telekom Laboratories. In: ISPIM-Asia Conference (2007)

10. Björk, J., Magnusson, M.: Where do good innovation ideas come from? Exploring the influence of network connectivity on innovation idea quality. J. Prod. Innov. Manag. 26(6), 662-670 (2009)

11. Kruse, P., Schieber, A., Hilbert, A., Schoop, E.: Idea mining-text mining supported knowledge management for innovation purposes. In: AMCIS (2013)

12. Rhyn, M., Blohm, I., Leimeister, J.M.: Understanding the emergence and recombination of distant knowledge on crowdsourcing platforms. In: 38th International Conference on Information Systems: Transforming Society with Digital Innovation, ICIS (2018)

13. Bloom, N., Jones, C.I., Van Reenen, J., Webb, M.: Are ideas getting harder to find? (w23782). National Bureau of Economic Research (2017)

14. Sandberg, A.B., Crnkovic, I.: Meeting industry-academia research collaboration challenges with agile methodologies. In: IEEE/ACM 39th International Conference on Software Engineering: Software Engineering in Practice Track (ICSE-SEIP), pp. 73-82 (2017)

15. Brijs, K.: Collaboration between academia and industry: KU Leuven. Cereal Foods World 62(6), 264-266 (2017)

16. Debortoli, S., Müller, O., Junglas, I.A., vom Brocke, J.: Text mining for information systems researchers: an annotated topic modeling tutorial. In: CAIS, vol. 39, no. 1, p. 7 (2016)

17. Kornish, L.J., Hutchison-Krupat, J.: Research on idea generation and selection: implications for management of technology. Prod. Oper. Manag. 26(4), 633-651 (2017)

18. Steingrimsson, B., Yi, S., Jones, R., Kisialiou, M., Yi, K., Rose, Z.: Big Data Analytics for Improving Fidelity of Engineering Design Decisions. SAE Technical Paper (2018)

19. Blei, D.M.: Probabilistic topic models. Commun. ACM 55(4), 77-84 (2012)

20. Wirth, R., Hipp, J.: CRISP-DM: towards a standard process model for data mining. In: Proceedings of the 4th International Conference on the Practical Applications of Knowledge Discovery and Data Mining, pp. 29-39 (2000)

21. Asamoah, D.A., Sharda, R.: Adapting CRISP-DM process for social network analytics: application to healthcare. AMCIS (2015) 
22. Ayele, W.Y.: Adapting CRISP-DM for idea mining: a data mining process for generating ideas using a textual dataset. Int. J. Adv. Comput. Sci. Appl. 11(6), 20-32 (2020)

23. Ayele, W.Y., Juell-Skielse, G.: Eliciting evolving topics, trends and foresight about self-driving cars using dynamic topic modeling. In: Arai, K., Kapoor, S., Bhatia, R. (eds.) FICC 2020. AISC, vol. 1129, pp. 488-509. Springer, Cham (2020). https://doi.org/10.1007/978-3-03039445-5_37

24. Ayele, W.Y., Akram, I.: Identifying emerging trends and temporal patterns about self-driving cars in scientific literature. In: Arai, K., Kapoor, S. (eds.) CVC 2019. AISC, vol. 944, pp. 355372. Springer, Cham (2020). https://doi.org/10.1007/978-3-030-17798-0_29

25. Liu, H., Goulding, J., Brailsford, T.: Towards computation of novel ideas from corpora of scientific text. In: Appice, A., Rodrigues, P.P., Santos Costa, V., Gama, J., Jorge, A., Soares, C. (eds.) ECML PKDD 2015. LNCS (LNAI), vol. 9285, pp. 541-556. Springer, Cham (2015). https://doi.org/10.1007/978-3-319-23525-7_33

26. Rohrbeck, R.: Trend scanning, scouting and foresight techniques. In: Gassmann, O., Schweitzer, F. (eds.) Management of the Fuzzy Front End of Innovation, pp. 59-73. Springer, Cham (2014). https://doi.org/10.1007/978-3-319-01056-4_5

27. Kao, S.C., Wu, C.H., Syu, S.W.: A creative idea exploration model: based on customer complaints. In: 5th MISNC (2018)

28. McIntosh, T., Mulhearn, T.J., Mumford, M.D.: Taking the good with the bad: the impact of forecasting timing and valence on idea evaluation and creativity. Psychol. Aesthet. Creat. Arts (2019)

29. Stevanovic, M., Marjanovic, D., Storga, M.: A model of idea evaluation and selection for product innovation. In: DS 80-8 Proceedings of the 20th International Conference on Engineering Design: Innovation and Creativity, vol. 15, no. 8, pp. 193-202 (2015)

30. Dean, D.L., Hender, J., Rodgers, T., Santanen, E.: Identifying good ideas: constructs and scales for idea evaluation. J. Assoc. Inf. Syst. 7(10), 646-699 (2006)

31. Saaty, T.L., Vargas, L.G.: Models, Methods, Concepts \& Applications of the Analytic Hierarchy Process. Springer, New York (2012). https://doi.org/10.1007/978-1-4614-3597-6

32. Afshari, A., Mojahed, M., Yusuff, R.M.: Simple additive weighting approach to personnel selection problem. Int. J. Innov. Manag. Technol. 1(5), 511 (2010)

33. Feldman, R., Sanger, J.: The Text Mining Handbook: Advanced Approaches in Analyzing Unstructured Data. Cambridge University Press. Cambridge (2007)

34. Hu, X., Liu, H.: Text analytics in social media. In: Aggarwal, C., Zhai, C. (eds.) Mining Text Data, pp. 385-414. Springer, Boston (2012). https://doi.org/10.1007/978-1-4614-3223-4_12

35. Bird, S., Klein, E., Loper, E.: Natural Language Processing with Python: Analyzing Text with the Natural Language Toolkit. O'Reilly Media, Inc. (2009)

36. Sidorov, G., Velasquez, F., Stamatatos, E., Gelbukh, A., Chanona-Hernández, L.: Syntactic n-grams as machine learning features for natural language processing. Expert Syst. Appl. 41(3), 853-860 (2014)

37. AlSumait, L., Barbará, D., Domeniconi, C.: On-line LDA: adaptive topic models for mining text streams with applications to topic detection and tracking. In: Eighth IEEE International Conference on Data Mining. ICDM 2008, pp. 3-12 (2008). IEEE

38. Blei, D.M., Lafferty, J.D.: Topic models. In Text Mining, pp. 101-124. Chapman and Hall/CRC (2009)

39. Blei, D.M., Lafferty, J.D.: Dynamic topic models. In: Proceedings of the 23rd International Conference on Machine Learning, pp. 113-120. ACM (2006)

40. Blei, D.M., Ng, A.Y., Jordan, M.I.: Latent dirichlet allocation. J. Mach. Learn. Res. 3, $993-$ $1022(2003)$ 
41. Keim, D., Andrienko, G., Fekete, J.-D., Görg, C., Kohlhammer, J., Melançon, G.: Visual analytics: definition, process, and challenges. In: Kerren, A., Stasko, John T., Fekete, J.D., North, C. (eds.) Information Visualization. LNCS, vol. 4950, pp. 154-175. Springer, Heidelberg (2008). https://doi.org/10.1007/978-3-540-70956-5_7

42. Chen, C., Hu, Z., Liu, S., Tseng, H.: Emerging trends in regenerative medicine: a scientometric analysis in CiteSpace. Expert Opin. Biol. Ther. 12(5), 593-608 (2012)

43. Alksher, M.A., Azman, A., Yaakob, R., Kadir, R.A., Mohamed, A., Alshari, E.: A framework for idea mining evaluation. In: SoMeT, pp. 550-559 (2017)

44. Dellermann, D., Lipusch, N., Li, M.: Combining humans and machine learning: a novel approach for evaluating crowdsourcing contributions in idea contests. In: Multikonferenz Wirtschaftsinformatik (2018)

45. Peffers, K., Tuunanen, T., Rothenberger, M.A., Chatterjee, S.: A design science research methodology for information systems research. J. Manag. Inf. Syst. 24(3), 45-77 (2007)

46. Aghaei, C.A., et al.: A comparison between two main academic literature collections: web of Science and Scopus databases. Asian Soc. Sci. 9(5), 18-26 (2013)

47. Small, H., Boyack, K.W., Klavans, R.: Identifying emerging topics in science and technology. Res. Policy 43(8), 1450-1467 (2014)

48. You, H., Li, M., Hipel, K.W., Jiang, J., Ge, B., Duan, H.: Development trend forecasting for coherent light generator technology based on patent citation network analysis. Scientometrics 111(1), 297-315 (2017)

49. Salatino, A.A., Osborne, F., Motta, E.: AUGUR: forecasting the emergence of new research topics. In: Proceedings of the 18th ACM/IEEE on Joint Conference on Digital Libraries, pp. 303-312 (2018)

50. Braun, V., Clarke, V.: Using thematic analysis in psychology. Qual. Res. Psychol. 3(2), 77-101 (2006)

51. Aggarwal, V., Hwang, E., Tan, Y.: Fostering innovation: Exploration is not everybody's cup of tea. In: 39th International Conference on Information Systems, ICIS (2018) 